\title{
Single-Use Autoinjector Functionality And
} Reliability For At-Home Administration Of

\section{Benralizumab For Patients With Severe Asthma: GRECO Trial Results}

This article was published in the following Dove Press journal:

Journal of Asthma and Allergy

\author{
Gary T Ferguson' \\ Jeremy Cole ${ }^{2}$ \\ Magnus Aurivillius ${ }^{3}$ \\ Paul Roussel ${ }^{4}$ \\ Peter Barker ${ }^{4}$ \\ Ubaldo J Martin (10) \\ On behalf of the GRECO \\ study investigators
}

'Pulmonary Research Institute of Southeast Michigan, Farmington Hills, MI, USA; ${ }^{2}$ OK Clinical Research, LLC, Edmond, OK, USA; ${ }^{3}$ AstraZeneca, Mölndal, Sweden; ${ }^{4}$ AstraZeneca, Gaithersburg, MD, USA
Correspondence: Gary T Ferguson Pulmonary Research Institute of Southeast Michigan, 29255 West 10 Mile

Road, Suite A, Farmington Hills, MI 48336, USA

Tel $+\mathrm{I}-248-478-6806$

$\mathrm{Fax}+\mathrm{I}-248-478-6908$

Email garytferguson@msn.com
Purpose: Accessorized prefilled syringes (APFS) have demonstrated functionality and reliability for subcutaneous (SC) delivery, including self-administration, of benralizumab $30 \mathrm{mg}$ in the clinic or at home. The multicenter, open-label GRECO study (NCT02918071) assessed functionality and reliability of a single-use autoinjector (AI) for at-home benralizumab administration by patients or their caregivers.

Patients and methods: Adults with severe asthma received benralizumab SC injections at the study site at Weeks 0,4 , and 8 . The first dose was administered by health care providers. Patients/caregivers had the option of administering the second dose and were required to administer the third dose under supervision. At Weeks 12 and 16, patients/caregivers administered benralizumab via $\mathrm{AI}$ at home. After each administration, patients/caregivers completed questionnaires concerning administration and device functioning. All AI devices used were returned for evaluation.

Results: A total of 595 AIs were used for 121 patients (mean age 48.5 years; 64\% female) in the clinic and at home. Of 116 participants, $113(97.4 \%$; 95\% confidence interval [CI]: 92.63-99.46) and 112 (96.6\%; 95\% CI: 91.41-99.05) successfully administered benralizumab at home at Weeks 12 and 16, respectively; 108 (93.1\%; 95\% CI: 86.86-96.98) were successful on both occasions. Throughout the study, $10(1.7 \%)$ AI administrations were unsuccessful: $8(1.3 \%)$ because of user error, $1(0.2 \%)$ with undetermined cause, and 1 $(0.2 \%)$ because of a manufacturing defect. Benralizumab efficacy (assessed by Asthma Control Questionnaire 6 score) and pharmacokinetics for patients using the AI were comparable to published results for patients receiving benralizumab via syringe in a clinical setting. No new or unexpected safety findings were observed.

Conclusion: AIs were functional, reliable, and performed well in the clinic and at home. Nearly all patients and caregivers successfully administered SC benralizumab via AI. Benralizumab availability in AI and APFS could provide patients with choices for self-administration.

Keywords: Autoinjector, benralizumab, biologic, clinical trial, delivery system, eosinophil, prefilled syringe, subcutaneous

\section{Plain Language Summary Why Was The Study Done?}

With some asthma medicines, patients need to see their doctors for every injection. We know patients can self-inject the asthma medicine benralizumab with prefilled syringes. We studied 
another option, autoinjectors (AI for short), which look like large pens (see Figure 1B). AIs are used for self-injections of many medicines. When activated, AIs inject medicine through a small needle.

\section{What Did The Researchers Do And Find?}

We studied AI use by patients with severe asthma and their caregivers. Patients received five injections of benralizumab with the AI. The first three injections were at a doctor's office. A doctor gave the first injection. Patients and caregivers had a choice for the second injection. They could use the AI while their doctors watched or have the doctors use the AI. Patients or caregivers used the AI for the third injection, then they used the $\mathrm{AI}$ at home for the next two injections.

Almost all patients and caregivers (93\%) successfully gave both at-home injections with the AI. Patients' blood had expected amounts of benralizumab after they used the AI, and asthma symptoms improved, meaning the medicine was working. Some patients had colds or other infections, asthma symptoms, or headaches during the study. Eight patients had pain, redness, or itching at the injection site. These were mild and lasted 1-8 days. No patients had allergic reactions to benralizumab.

A

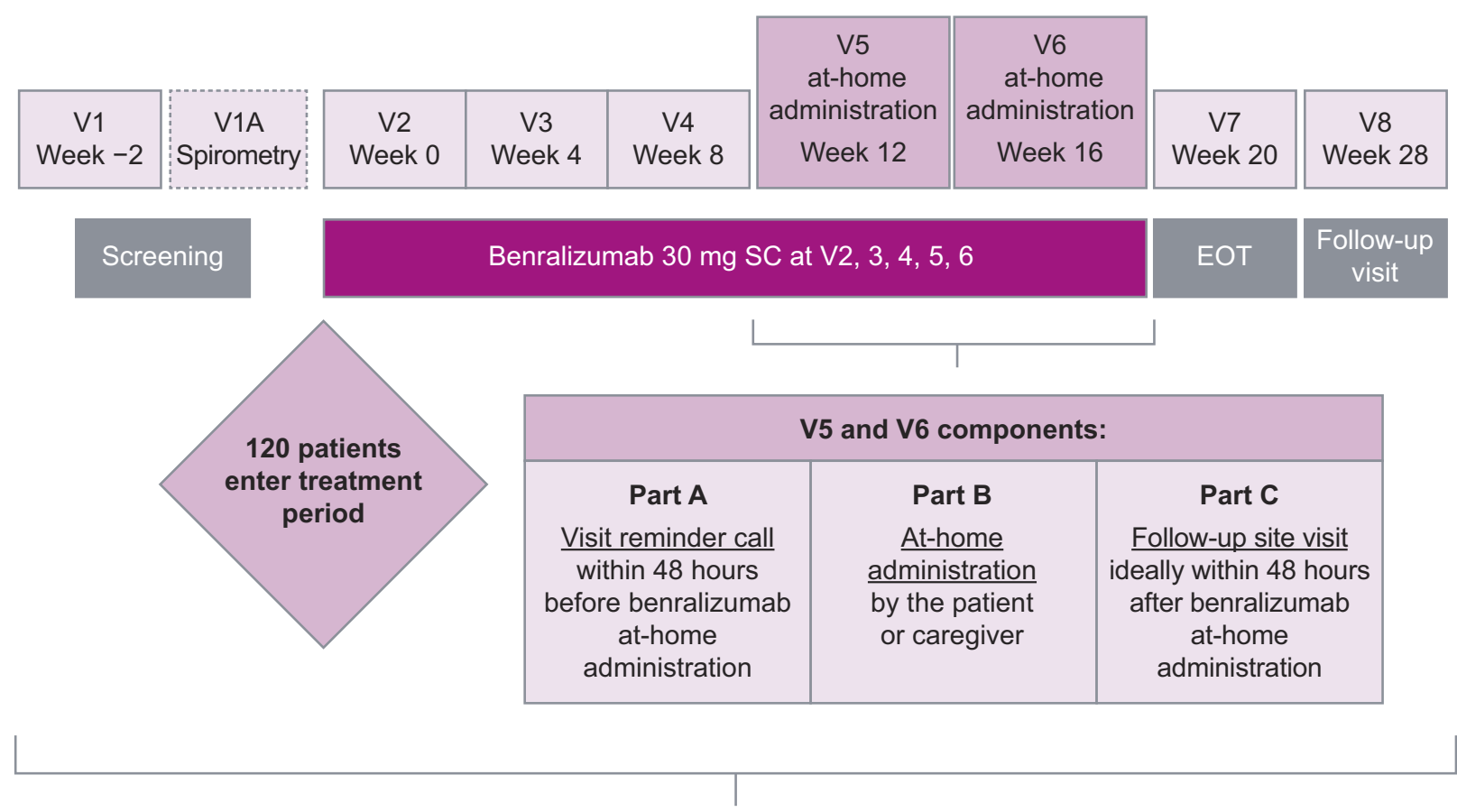

Total study duration: 30 weeks

B

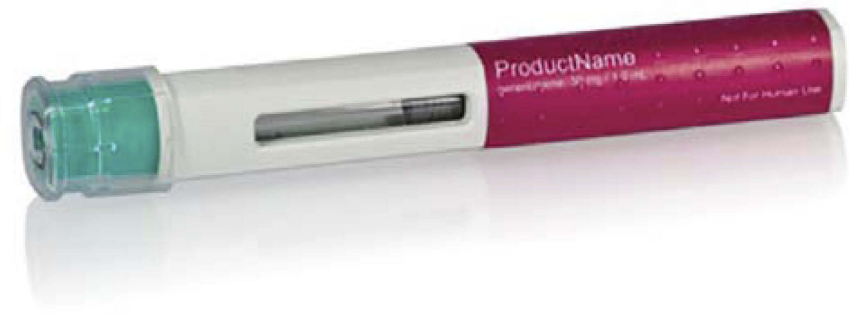

Figure I GRECO study design (A) and single-use autoinjector (B).

Abbreviations: EOT, end of treatment; SC, subcutaneously; $\mathrm{V}$, visit. 


\section{What Do These Results Mean?}

Patients and caregivers can use AIs to inject benralizumab. AIs and prefilled syringes offer more choices for patients receiving benralizumab for severe asthma and allow patients to self-inject at places such as home.

\section{Introduction}

Eosinophilic asthma is often severe, with patients experiencing increased exacerbation frequency, impaired lung function, and poor asthma control. ${ }^{1-3}$ Benralizumab is a humanized, afucosylated monoclonal antibody that binds to the alpha subunit of the human interleukin-5 receptor on the surface of blood eosinophils with high affinity and specificity. This leads to nearly complete depletion of blood and tissue eosinophils through enhanced antibodydependent cell-mediated cytotoxicity, an apoptotic process involving immune effector cells such as natural killer cells and macrophages. ${ }^{4,5}$ Benralizumab significantly reduces annual asthma exacerbation rates and oral corticosteroid use and improves lung function and disease-specific patient-reported outcomes for patients with blood eosinophil counts $\geq 150$ cells $/ \mu \mathrm{L}$. $^{6-9}$ Like all currently available biologic treatments for asthma, benralizumab is an injectable medication. Patients now receive benralizumab $30 \mathrm{mg}$ as a subcutaneous injection in a clinical setting every 8 weeks (first three doses every 4 weeks). However, many patients prefer at-home self-administration of injectable medications to administration by health care professionals in a clinical setting. ${ }^{10-12}$

Options for administration of injectable medications include single-use accessorized prefilled syringes (APFS) and autoinjectors (AI). APFS have demonstrated functionality for administration of fixed doses of biologics for the treatment of allergic asthma or severe, uncontrolled eosinophilic asthma. ${ }^{13,14}$ In a study of benralizumab selfadministration, most patients and caregivers ( $\geq 98 \%)$ used APFS successfully. ${ }^{14}$ Of 573 APFS used, one malfunction and two user errors resulted in unsuccessful at-home administrations. Injection-site reactions were mild to moderate and resolved in 1-15 days. ${ }^{14}$ Efficacy, pharmacokinetic, and pharmacodynamic assessments confirmed that benralizumab exposure following at-home use of APFS was comparable to exposure following administration by health care professionals at clinical sites. ${ }^{14}$ A further study of benralizumab with healthy volunteers demonstrated that pharmacokinetics following a single injection were similar between APFS and AI. ${ }^{15}$
The aim of the current study was to assess patient- or caregiver-reported functionality and reliability of AI for benralizumab administration, including self-administration, in a home setting. This study also assessed efficacy and pharmacokinetics of benralizumab administered via AI.

\section{Materials And Methods Study Design And Population}

GRECO (NCT02918071) was a multicenter, nonrandomized, open-label Phase III trial conducted at 25 sites in the United States and Canada (Supplemental Table 1). The study included a 2-week screening period, a site-administration treatment period (Weeks 0-8), a home-administration treatment period (Weeks 12-16), an end-of-treatment visit (Week 20), and a follow-up visit (Week 28; Figure 1A).

Eligible patients were men and women aged 18-75 years who had severe, uncontrolled asthma and weighed $\geq 40 \mathrm{~kg}$. Evidence of asthma was defined as airway reversibility based on history and an increase in postbronchodilator forced expiratory volume in 1 second $\left(\mathrm{FEV}_{1}\right)$ of $\geq 12 \%$ and $\geq 200 \mathrm{~mL}$ at screening or first treatment visit. Patients were also required to have documented history of current treatment with inhaled corticosteroids and long-acting $\beta_{2^{-}}$ agonists, morning prebronchodilator $\mathrm{FEV}_{1}>50 \%$ predicted normal at screening or first treatment visit, and asthma that was "not well-controlled." Not-well-controlled asthma was defined as an Asthma Control Questionnaire 6 (ACQ-6) score $\geq 1.5$, peak expiratory flow $60-80 \%$ predicted normal, or $\geq 1$ exacerbation requiring oral or systemic corticosteroids in the previous year. Exclusion criteria included clinically important pulmonary diseases other than asthma; medical disorders that, in the opinion of the investigator, were not stable and could affect patient safety, study results, or patient completion of the trial; drug or alcohol abuse in the previous year; and status as a current smoker or smoking history of $\geq 10$ pack-years.

\section{Ethics}

An independent ethics committee or institutional review board for each study center approved the final study protocol. All patients provided written informed consent prior to study initiation. This study was performed in accordance with the Declaration of Helsinki, International Council for Harmonisation/Good Clinical Practice guidelines, the 
applicable regulatory requirements, and AstraZeneca policy on bioethics.

\section{Data-Sharing Statement}

Data underlying the findings described in this manuscript may be requested in accordance with AstraZeneca's data sharing policy described at https://astrazenecagroup-dt. pharmacm.com/DT/Home.

\section{Procedures}

Benralizumab (manufactured by AstraZeneca, Gaithersburg, MD, USA) was administered as a $30-\mathrm{mg} / \mathrm{mL}$ solution in a 1$\mathrm{mL}$ single-use AI device (Figure 1B). Patients received openlabel benralizumab during study site visits at Weeks 0 , 4, and 8 . At Week 0 , benralizumab was administered by a health care professional via the AI. At Week 4, patients/caregivers had the option of allowing a health care professional to administer benralizumab via the $\mathrm{AI}$ or using the $\mathrm{AI}$ themselves under the supervision of a health care professional. At Week 8, patients/ caregivers were required to use the AI to administer benralizumab under the supervision of a health care professional. Patients received written instructions on AI use for reference at home. At Weeks 12 and 16, patients/caregivers administered benralizumab at home via the $\mathrm{AI}$ and completed a questionnaire on functionality and administration.

After each at-home administration, patients/caregivers returned for a study visit within $48 \mathrm{hrs}$, bringing the questionnaire and used AI with them. Study sites returned the used AI to the study sponsor for evaluation of functionality.

\section{Outcomes}

The primary objective was to assess patient- or caregiverreported functionality and reliability of the AI device in an at-home setting and the performance of the AI after use. Outcome measures for this objective were the percentage of patients/caregivers who successfully administered benralizumab at home via the AI device, percentage of AI devices used to administer benralizumab at home that were considered functional, and percentage of AI devices used to administer benralizumab that were considered malfunctioning (at home or in the clinic). Secondary endpoints were change from baseline in mean ACQ-6 score, benralizumab pharmacokinetics and immunogenicity, and change in blood eosinophil counts. Safety endpoints included assessment of adverse events (AEs) and serious AEs.

Successful benralizumab administration via the AI was defined as a completed injection, answers of "yes" to all five questionnaire items, and satisfactory examination of the returned device after use. The questionnaire asked (yes/no) whether patients/caregivers could 1) view the liquid through the AI viewing window, 2) start the injection, 3) hold the AI in place until the injection was completed, 4) see the blue plunger in the viewing window after injection, and 5) confirm the needle guard slid down to cover the needle following injection. A central quality control unit examined the AI devices after use and conducted a visual inspection to assess physical integrity, presence of any disassembled components, whether the plunger was able to completely depress to deliver a full dose, and whether the needle guard fully deployed. The central control unit also tested the needle guard to ensure the safety mechanism stayed locked in place when pressure was applied. Serum benralizumab concentration and the presence of serum antidrug antibodies (ADA) were measured predose (Week 0 ) and at Weeks 8, 20, and 28. Blood eosinophil counts were measured as part of the routine hematology assessments at Weeks 0, 20, and 28.

The ACQ-6 is a shortened version of the ACQ that assesses asthma symptoms (night-time awakenings, symptoms on awakening, activity limitation, shortness of breath, and wheezing) and short-acting $\beta_{2}$-agonist use, omitting the $\mathrm{FEV}_{1}$ measurement from the original ACQ. ${ }^{16}$ For each item, patients were asked to rate their experiences during the previous week on a 7-point scale ranging from 0 (totally controlled) to 6 (severely uncontrolled). Answers to all items were averaged to determine overall ACQ-6 score. Scores $\leq 0.75$ indicated well-controlled asthma, scores $>0.75$ and $<1.5$ indicated partially controlled asthma, and scores $\geq 1.5$ indicated poorly controlled asthma. ${ }^{16}$ Decreasing ACQ-6 scores indicated improving asthma symptoms. A decrease of 0.5 in ACQ6 score was considered to indicate clinically important improvement.

\section{Statistical Analysis}

No hypotheses were tested statistically. An estimated sample size of 120 patients was based on a target of 100 patients completing the study, with an estimated dropout rate of $17 \%$ (20 patients). Summary data were presented in tabular format, and categorical data are summarized by the number and percentage of patients in each category. Continuous data are summarized by descriptive statistics including number, mean, standard deviation (SD), median, and range. Change from baseline was computed as 
baseline value subtracted from visit value. If either value was missing, change from baseline was set to missing.

All analyses were performed with the full analysis set except the pharmacokinetic analysis, which used the pharmacokinetic analysis set. The full analysis set included all patients receiving benralizumab, regardless of their protocol adherence and continued participation in the study. Patients with at least one quantifiable serum benralizumab concentration after the first dose were included in the pharmacokinetic analysis set. All data were analyzed with SAS System version 9.2 (SAS Institute Inc., Cary, NC, USA).

\section{Results}

\section{Patient Disposition}

Patients were screened at 25 centers and received treatment at 23 centers. Of 121 patients who received at least one dose of benralizumab, five (4.1\%) discontinued treatment during the study (Figure 2). Four patients discontinued before athome administration via the AI for the following reasons: AE (Herpes zoster), lost to follow up, inability to complete Visit 4 within the study time frame, and development of study-specific discontinuation criteria (patient unwilling to administer benralizumab at home). One patient discontinued after one successful at-home administration because of a decision to discontinue birth control.

\section{Demographics And Baseline Clinical Characteristics}

Demographics and baseline clinical characteristics represented the intended target population of patients with uncontrolled asthma (Table 1). Mean age was 48.5 years (SD: 14.95), and median body mass index was $30.4 \mathrm{~kg} / \mathrm{m}^{2}$ (range: $20-64 \mathrm{~kg} / \mathrm{m}^{2}$ ). Most patients were white $(80.2 \%$ ), and nearly two-thirds were female (63.6\%). Median time since asthma diagnosis was 20.0 years (range: $0.1-61.0$ years). Mean percentage predicted normal prebronchodilator $\mathrm{FEV}_{1}$ was $69.3 \%$ (SD: 12.7\%). Mean baseline ACQ-6 score was 2.42 (SD: 0.96), which was consistent with expected values for patients with uncontrolled asthma. Mean number of exacerbations in the past 12 months was 0.8 (SD: 1.5); approximately half of patients $(53.7 \%)$ had none. Other key respiratory diseases included allergic rhinitis $(72.7 \%$ of patients) and nasal polyps (10.7\% of patients).

\section{Functionality, Reliability, And Performance Of Autoinjectors}

\section{Study-Site Administration}

Benralizumab was successfully administered at the study site to 121 patients (100\%) at Week 0. At Week 4, 119 of 121 administrations were successful. Both unsuccessful administrations were performed by patients. One patient

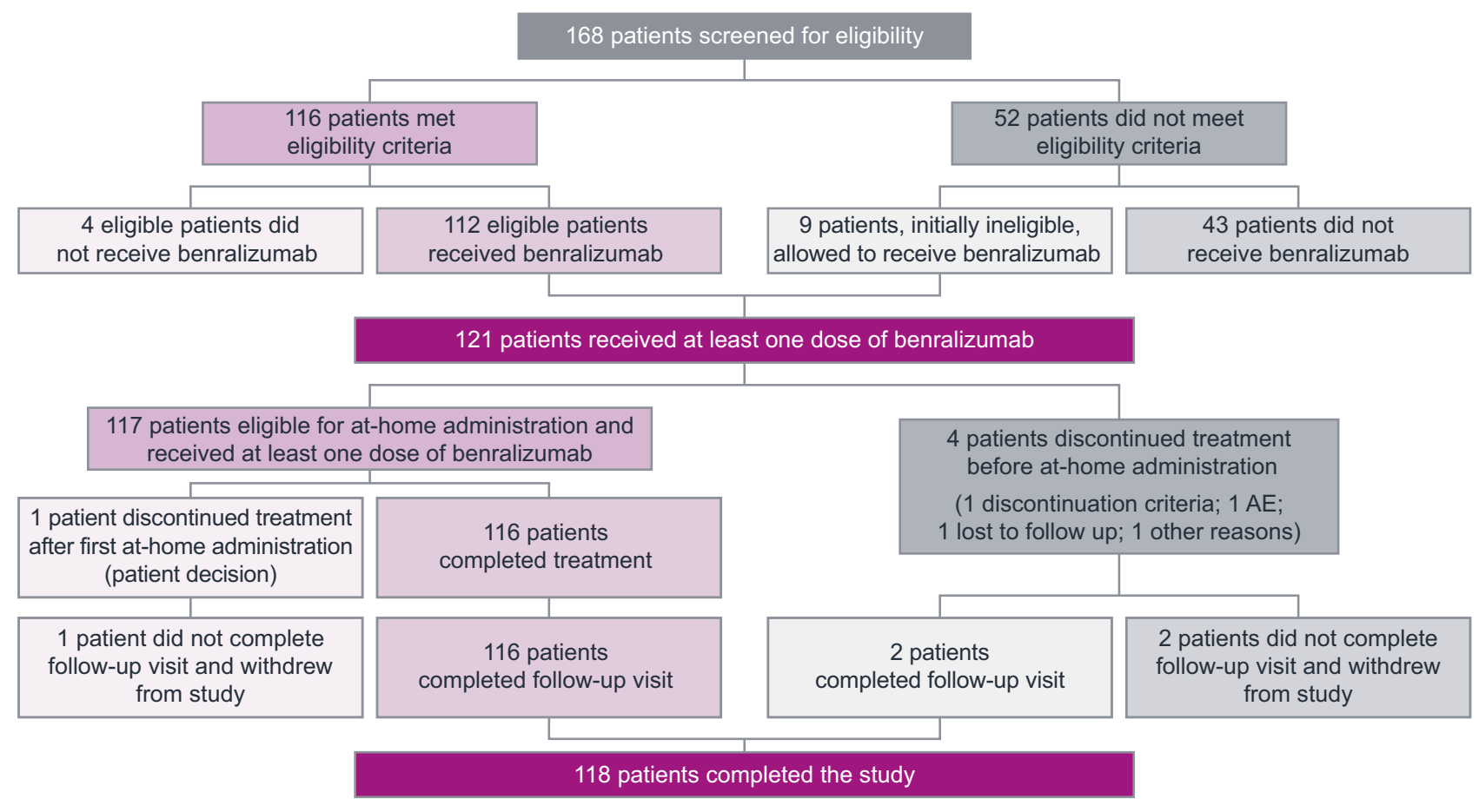

Figure 2 Patient flow diagram.

Abbreviation: $\mathrm{AE}$, adverse event. 
Table I Demographics And Baseline Clinical Characteristics

\begin{tabular}{|c|c|}
\hline Demographics/Clinical Characteristics & $\begin{array}{l}\text { Benralizumab } \\
30 \mathrm{mg} \\
\mathrm{N}=12 \mathrm{I}\end{array}$ \\
\hline Age [years], mean (SD) & $48.5(14.95)$ \\
\hline Age group [years], n (\%) & \\
\hline$\geq 18$ to $<50$ & $54(44.6)$ \\
\hline$\geq 50$ to $<65$ & $50(4 I .3)$ \\
\hline$\geq 65$ & $17(14.0)$ \\
\hline Sex, n (\%) & \\
\hline Female & $77(63.6)$ \\
\hline Race, n (\%) & \\
\hline White & $97(80.2)$ \\
\hline Black or African-American & $14(11.6)$ \\
\hline Asian & $9(7.4)$ \\
\hline Other & $\mathrm{I}(0.8)$ \\
\hline Ethnic group, n (\%) & \\
\hline Hispanic or Latino & $26(21.5)$ \\
\hline BMI $\left[\mathrm{kg} / \mathrm{m}^{2}\right]$, median (range) & $30.4(20-64)$ \\
\hline Blood eosinophil count [cells/ $\mu \mathrm{L}]$, median (IQR) & $230(150-360)$ \\
\hline Prebronchodilator FEV, [L], mean (SD) & $2.126(0.676)$ \\
\hline Prebronchodilator FEV , [\% PN], mean (SD) & $69.3(12.7)$ \\
\hline Prebronchodilator FVC [L], mean (SD) & $3.097(1.008)$ \\
\hline Prebronchodilator FVC [\% PN], mean (SD) & $81.0(15.2)$ \\
\hline FEV, reversibility [\%], mean (SD) & $20.0(10.0)$ \\
\hline Time since asthma diagnosis [years], median (range) & $20.0(0.1-61.0)$ \\
\hline ACQ-6 score, mean (SD) & $2.42(0.96)$ \\
\hline $\begin{array}{l}\text { Exacerbations in the previous } 12 \text { months, mean } \\
\text { (SD) }\end{array}$ & $0.8(1.5)$ \\
\hline $0, \mathrm{n}(\%)$ & $65(53.7)$ \\
\hline I, n (\%) & $40(33.1)$ \\
\hline $2, \mathrm{n}(\%)$ & $6(5.0)$ \\
\hline $3, \mathrm{n}(\%)$ & $7(5.8)$ \\
\hline$\geq 4, \mathrm{n}(\%)$ & $3(2.5)$ \\
\hline \multicolumn{2}{|l|}{$\begin{array}{l}\text { Number of exacerbations in the last } 12 \text { months } \\
\text { resulting in: }\end{array}$} \\
\hline Hospitalization, mean (SD) & $0.1(0.3)$ \\
\hline ED visit, mean (SD) & $0.1(0.4)$ \\
\hline Diagnosis of allergic rhinitis, $n(\%)$ & $88(72.7)$ \\
\hline Nasal polyps, n (\%) & $13(10.7)$ \\
\hline OCS use, n (\%) & $4(3.3)$ \\
\hline
\end{tabular}

Abbreviations: ACQ-6, Asthma Control Questionnaire 6; BMI, body mass index; $E D$, emergency department; $\mathrm{FEV}_{1}$, forced expiratory volume in I second; FVC, forced vital capacity; IQR, interquartile range; OCS, oral corticosteroid; PN, predicted normal; SD, standard deviation. did not keep the AI pressed firmly against the injection site for the entire injection. The other patient lifted the AI away from the skin before the injection was complete. Both patients completed the study, and all their subsequent administrations were successful. At Week 8, 118 of 119 administrations were successful. The single unsuccessful administration occurred because the patient did not keep the AI pressed firmly against the injection site for the entire injection. This patient completed the study and was able to perform all subsequent injections successfully.

\section{At-Home Administration}

One AI used at home at Week 12 was not returned by the patient and could not be inspected after use. Of 116 patients/caregivers who administered benralizumab at home at Week 12 and returned their devices, 113 (97.4\%; 95\% confidence interval [CI]: 92.63-99.46) were successful (Figure 3). The three unsuccessful administrations were caused by lifting the AI away from the skin before the injection was complete, prematurely activating the AI during removal of the cap, and the need to manually retract the needle guard on the device. Once the needle guard was retracted, the patient was able to inject the full dose. All three patients with unsuccessful administrations at Week 12 completed the study, and all their subsequent administrations were successful. At Week 16, 112 (96.6\%;

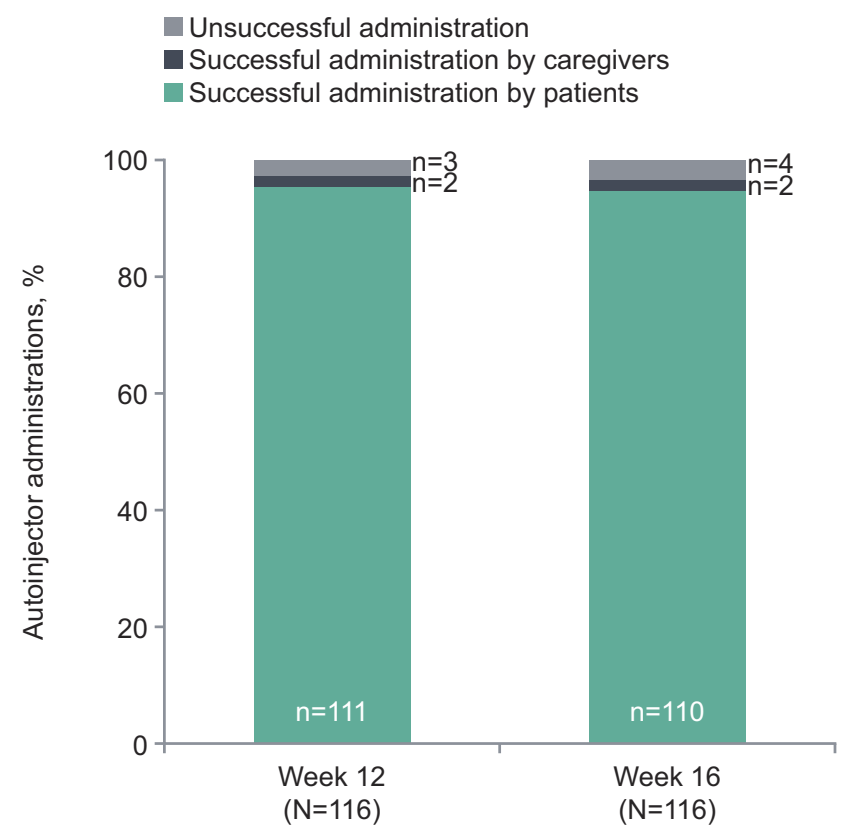

Figure 3 Success of at-home benralizumab administration via autoinjector. Successful administration was defined as a completed injection, an answer of "yes" to all five questions in the administration questionnaire, and satisfactory evaluation of returned devices. 
95\% CI: 91.41-99.05) of 116 patients/caregivers used the AI successfully. Unsuccessful administrations were caused by delayed retraction of the needle guard, possible premature activation of the AI, removing the AI from the skin before completion of the injection, and losing control of the AI device. Most patients were able to self-administer both benralizumab injections at home $(93.8 \%$; 95\% CI: 87.65-97.47). All caregivers (100\%; 95\% CI: 15.81100.00) successfully completed both at-home benralizumab administrations with the AI.

The two patients who prematurely activated their AI devices (one at Week 12 and one at Week 16) received replacements. These patients returned both their original devices and the replacements. Hence, the number of AI following both the Week 12 and Week 16 at-home benralizumab administrations was 117 , one more than the number of patients assessed. Of the AI returned after Weeks 12 and 16, 114 (97.4\%) and 113 (96.6\%), respectively, were deemed functional by patients/caregivers. All returned AI were also evaluated for functionality by a central quality unit. Of 595 AI used in this study, 10 (1.7\%) administrations were unsuccessful: three at the study site and seven at home. Examination by the quality unit indicated that two AI malfunctioned (one because of a manufacturer defect). The root cause of the other could not be definitively identified. Unsuccessful administration with the remaining eight AI were determined to be caused by user error.

\section{Benralizumab Efficacy, Pharmacokinetics, Pharmacodynamics, And Immunogenicity}

By the end of treatment (Week 20), mean ACQ-6 score decreased from baseline, and approximately one-third of patients had asthma that was well-controlled (Figure 4, Table 2). The anti-IL-5 receptor alpha properties of benralizumab were evident in that nearly complete depletion of blood

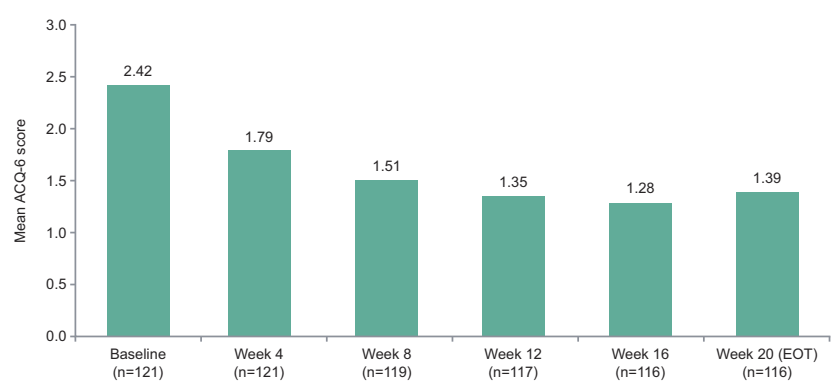

Figure 4 Mean ACQ-6 score by time point. ACQ-6 score is the average of the first six items on the ACQ regarding symptoms, activity limitations, and rescue medication use.

Abbreviations: ACQ-6, Asthma Control Questionnaire 6; EOT, end of treatment.
Table 2 Asthma Control Status Based On ACQ-6 Score At Baseline And End Of Treatment

\begin{tabular}{|l|l|}
\hline & $\begin{array}{l}\text { Benralizumab } \\
(\mathbf{N}=121)\end{array}$ \\
\hline Baseline, $\mathrm{n}(\%)$ & \\
$\leq 0.75$ (well-controlled) & $5(4.1)$ \\
$>0.75$ to $<1.5$ (partly controlled) & $6(5.0)$ \\
$\geq 1.5$ (not well-controlled) & $110(90.9)$ \\
Missing, $\mathrm{n}$ (\%) & 0 \\
\hline End of treatment (Week 20), n (\%) & \\
$\leq 0.75$ (well-controlled) & $40(33.1)$ \\
$>0.75$ to <1.5 (partly controlled) & $23(19.0)$ \\
$\geq 1.5$ (not well-controlled) & $53(43.8)$ \\
Missing & $5(4.1)$ \\
\hline
\end{tabular}

Abbreviation: ACQ-6, Asthma Control Questionnaire 6.

eosinophils occurred by Week 20 (median: 20 cells $/ \mu \mathrm{L}$; interquartile range: $10-30$ cells $/ \mu \mathrm{L}$ ) and was maintained to Week 28 (median: 20 cells $/ \mu \mathrm{L}$; interquartile range: $10-50$ cells $/ \mu \mathrm{L}$ ). Geometric mean serum benralizumab concentrations were $698 \mathrm{ng} / \mathrm{mL}$ at Week 8, $788 \mathrm{ng} / \mathrm{mL}$ at Week 20, and $63 \mathrm{ng} /$ $\mathrm{mL}$ at Week 28 (Table 3).

No patients were ADA positive at baseline. However, $7.5 \%$ of patients ( 9 of 120) developed ADA after treatment began. Thus, both the prevalence and incidence of ADA were $7.5 \%$. The presence of neutralizing antibodies did not alter the pharmacodynamics of benralizumab, as demonstrated by comparable blood eosinophil counts at Week 20 for ADA-positive and ADA-negative patients (both median: 20 cells $/ \mu \mathrm{L})$.

\section{Safety Of Subcutaneous Benralizumab 30 Mg Administered Via Autoinjectors}

Benralizumab was well-tolerated, and mean duration of exposure was 110 days (range: 27-148 days). Of 121 patients, $74(61.2 \%)$ had at least one AE during the study (Table 4). Most AEs were mild to moderate. Four patients (3.3\%) experienced severe AEs: asthma (three patients) and retinal detachment (one patient). One incidence of asthma was also considered a serious AE. No severe or serious AEs were considered related to study drug or the AI device. One patient discontinued treatment because of an AE of Herpes zoster that was considered related to the study drug. The most common AEs (reported for $>5 \%$ of patients), were viral upper respiratory tract infection, asthma, upper respiratory tract infection, and headache (Table 4). Mild systemic AEs that the investigators considered to be related to benralizumab administration occurred for 13 patients (10.7\%): 
Table 3 Serum Benralizumab Concentrations

\begin{tabular}{|c|c|c|c|c|}
\hline & \multicolumn{4}{|l|}{ Visit } \\
\hline & Baseline $(n=120)$ & Week $8(n=|| 8)$ & Week $20(n=|| 5)$ & Week $28(n=\mid 17)$ \\
\hline $\mathrm{n}<$ LLOQ & 113 & 3 & 3 & 17 \\
\hline Geometric mean [ng/mL] (CV [\%]) & BLQ & $698(186)$ & $788(28 I)$ & $63(469)$ \\
\hline Arithmetic mean $[\mathrm{ng} / \mathrm{mL}](\mathrm{SD})$ & $18(116)$ & $1,000(663)$ & $\mathrm{I}, 24 \mathrm{I}(747)$ & $|5|(147)$ \\
\hline Median [ng/mL] (range) & $\begin{array}{l}\text { BLQ } \\
(<L L O Q-1,020)\end{array}$ & $\begin{array}{l}877 \\
(<L L O Q-3,890)\end{array}$ & $\begin{array}{l}\mathrm{I}, \mathrm{I} 70 \\
(<\mathrm{LLOQ}-3,330)\end{array}$ & $\begin{array}{l}106 \\
(<L L O Q-640)\end{array}$ \\
\hline
\end{tabular}

Abbreviations: BLQ, below limit of quantification; CV, coefficient of variation; LLOQ, lower limit of quantification (3.86 ng/mL); SD, standard deviation.

Table 4 AEs During The Study Period

\begin{tabular}{|l|l|}
\hline AEs, $\mathbf{n}$ (\%) & $\begin{array}{l}\text { Benralizumab } 30 \mathbf{~ m g} \\
(\mathbf{N}=12 \text { I) }\end{array}$ \\
\hline Patients with $\geq I$ serious AE & $\mathrm{I}(0.8)^{\mathrm{a}}$ \\
\hline Patients with $\geq I \mathrm{I} E^{\mathrm{b}}$ & $74(61.2)$ \\
\hline AEs experienced by $\geq 5 \%$ of patients: & \\
Viral upper respiratory tract infection & $18(14.9)$ \\
Asthma & $\mathrm{II}(9.1)$ \\
Upper respiratory tract infection & $10(8.3)$ \\
Headache & $8(6.6)$ \\
\hline
\end{tabular}

Notes: ${ }^{\text {aThis }}$ patient experienced asthma as a serious $A E$. ${ }^{b}$ Patients with $>I A E$ in the same category were counted only once in that category, and patients with AEs in $>$ I category were counted once in each category.

Abbreviation: $\mathrm{AE}$, adverse event.

two patients each experienced administration-site pruritus, fatigue, injection-site induration, injection-site pain, and injection-site pruritis; one patient each experienced administration-related reaction, headache, Herpes zoster, injection-site erythema, and inflammation. Injection-site reactions were reported by eight patients (6.6\%); all were mild and resolved in 1-8 days.

\section{Discussion}

The GRECO study evaluated the functionality, reliability, and performance of AIs for at-home administration, including self-administration, of benralizumab for patients with severe, uncontrolled asthma. AIs performed well in the clinic and at home and were successfully used for subcutaneous administration of benralizumab by health care providers, patients, and caregivers.

Only one manufacturer defect was detected in an AI, and one AI had a defect for which the root cause could not be identified. Eight administrations via AI failed because of user error. The most common error was lifting the AI away from the skin before the injection was complete.
Incidence of such errors could be reduced with additional training or instruction. Overall results of this study indicate that health care providers and almost all patients and caregivers were adequately trained to administer benralizumab subcutaneously via AI in the clinic or at home.

The mean 1-point improvement in ACQ-6 score during this 28-week study is within the range of improvements observed over 1 year in the SIROCCO and CALIMA studies and over 20 weeks in GREGALE, and indicates clinically important improvement. ${ }^{6,9,14}$ Furthermore, a nearly complete depletion of blood eosinophils after benralizumab 30-mg injection was observed in GRECO, similar to results in the GREGALE trial and in AMES, a pharmacokinetic study of single-dose benralizumab administration in a clinical setting. ${ }^{6,8,9,14,15}$ Pharmacokinetics of benralizumab administered via AI were stable during treatment, similar to that observed with APFS in the GREGALE study. ${ }^{14}$ These similarities confirm that patient and caregiver use of AI at home resulted in benralizumab exposure comparable to that observed in other clinical trials.

The safety profile of benralizumab administered via AI in this study was comparable to safety profiles from published clinical trials of benralizumab administered by health care providers at clinical sites and from the GREGALE study of benralizumab administered via APFS in both clinical and at-home settings. ${ }^{6,8,9,14,17}$ The incidence of AEs related to injection site in GRECO (6.6\% of patients) was numerically greater than that observed in the 12-week BISE and 28-week ZONDA studies (up to 3\% of patients), 28week GREGALE study (4\% of patients), and 1-year SIROCCO and CALIMA studies (both 2\%). ${ }^{8,14,17}$ In AMES, incidence of injection-site reactions was even smaller ( $1 \%$ for AI, none reported for APFS). ${ }^{15}$

Our results are consistent with those of studies in other therapeutic areas concerning self-administration of biologic treatments via AI. ${ }^{18-22}$ Pharmacokinetics, efficacy, and 
safety were comparable when biologics were administered via AI or syringes. ${ }^{18,19,22}$ Patients had high success rates when self-administering biologic treatments via AI. ${ }^{20,21}$ Studies also reported that patients viewed AI favorably and found them easy to use. ${ }^{22,23}$

The ability of patients to self-administer biologic asthma medications in an unsupervised setting may raise concerns regarding the potential for nonadherence to treatment. Physicians should certainly use their clinical judgment regarding which patients should continue to be treated in the clinic and which patients would do well with at-home administration. Endocrinologists, rheumatologists, gastroenterologists, and dermatologists have been prescribing biologics for at-home injection for many years. The best-known example, insulin, has been in use for nearly 100 years. ${ }^{24}$ At-home self-administration has the potential to reduce the burden on specialty clinics and allow physicians time to treat more patients with severe asthma. This would be especially important in medically underserved areas. For many patients, the convenience of at-home self-administration every 8 weeks could potentially improve adherence, especially where local clinic access is limited. Patients who live far from a treatment center or who find it difficult to make time for appointments would be among the potential candidates for athome self-administration.

A limitation of this study is the inclusion of only adult patients and their caregivers, as benralizumab is indicated for patients aged 12 years or older with severe eosinophilic asthma. We cannot exclude the possibility that other user errors or issues would have been observed with adult caregivers using AI to administer benralizumab to patients aged 12-18 years with asthma. Patients in this study received the same treatment regimen as was used in the GREGALE study (four doses of benralizumab $30 \mathrm{mg}$ at 4 -week intervals $)^{14}$ for best comparison of results between the two studies. However, the approved regimen for treatment of severe, uncontrolled eosinophilic asthma is benralizumab $30 \mathrm{mg}$ every 8 weeks (first three doses every 4 weeks). It should be noted that this was not a study of benralizumab safety or efficacy. We would not expect the difference in dosing frequency to have any influence on the functionality of AIs for athome administration. An interval of 8 weeks rather than 4 weeks between the two at-home administrations could potentially have resulted in differences in the number or type of user errors observed.

\section{Conclusions}

The use of AI at home or in the clinic is a well-tolerated and reliable method for administering subcutaneous benralizumab $30 \mathrm{mg}$ as an add-on treatment for patients with severe, uncontrolled asthma. Providing options for benralizumab self-administration (in a clinical setting or at home) may improve patient satisfaction and treatment adherence. Giving patients the opportunity to choose how, when, and where they receive their asthma treatments could play a substantial role in their treatment outcomes and overall experience.

\section{Abbreviations}

ACQ-6, Asthma Control Questionnaire 6; ADA, anti-drug antibodies; AE, adverse event; AI, autoinjectors; APFS, accessorized prefilled syringes; CI, confidence interval; $\mathrm{FEV}_{1}$, forced expiratory volume in 1 second; IL, interleukin; SD, standard deviation.

\section{Acknowledgments}

We thank the investigators, health care providers, research staff, and patients who participated in the GRECO study. We thank Mark Odorisio (AstraZeneca, Gaithersburg, MD) and Melanie Sundsbo Hughes (AstraZeneca, Gaithersburg, MD) for their clinical operations leadership in this study. Editorial support was provided by Jennie G. Jacobson, PhD, CMPP, of JK Associates, Inc., and Michael A. Nissen, ELS, of AstraZeneca.

\section{Author Contributions}

Gary T. Ferguson and Ubaldo J. Martin designed the study. All authors contributed to the clinical work for the study. All authors contributed to data analysis, drafting and revising the article critically for important intellectual content, gave final approval of the version to be published, and agree to be accountable for all aspects of the work.

\section{Disclosure}

AstraZeneca employees contributed to the design of the trial, the conduct of the trial, the data analysis and interpretation, and the writing of the manuscript. AstraZeneca funded medical writing support for the writing and submission of the manuscript. The GRECO trial was funded by AstraZeneca. Gary T Ferguson reports grants, personal fees, and nonfinancial support from AstraZeneca during the conduct of the study; grants, personal fees, and nonfinancial support from Boehringer Ingelheim, Novartis, 
AstraZeneca, Pearl Therapeutics (a member of the AstraZeneca group), and Sunovion; grants and personal fees from Theravance; and personal fees from Verona, Mylan, Innoviva, GlaxoSmithKline, and Circassia, outside the submitted work. Magnus Aurivillius, Paul Roussel, Peter Barker, and Ubaldo $\mathrm{J}$ Martin are employees of AstraZeneca. Jeremy Cole reports no conflicts of interest. Some of these data were presented in a poster at the American Academy of Allergy, Asthma and Immunology (AAAAI) Annual Meeting, February 22-25, 2019, San Francisco, CA, USA as a poster presentation. The poster abstract was published in The Journal of Allergy and Clinical Immunology: https://www.jacionline. org/article/S0091-6749(18)32034-7/fulltext.

The authors report no other conflicts of interest in this work.

\section{References}

1. Belda J, Parameswaran K, Lemiere C, Kamada D, O'Byrne PM, Hargreave FE. Predictors of loss of asthma control induced by corticosteroid withdrawal. Can Respir J. 2006;13(3):129-133. doi:10.1155/2006/189127

2. Price D, Wilson AM, Chisholm A, et al. Predicting frequent asthma exacerbations using blood eosinophil count and other patient data routinely available in clinical practice. J Asthma Allergy. 2016;9:112. doi:10.2147/JAA.S97973

3. Talini D, Novelli F, Bacci E, et al. Sputum eosinophilia is a determinant of $\mathrm{FEV}_{1}$ decline in occupational asthma: results of an observational study. BMJ Open. 2015;5(1):e05748. doi:10.1136/bmjopen2014-005748

4. Pham TH, Damera G, Newbold P, Ranade K. Reductions in eosinophil biomarkers by benralizumab in patients with asthma. Respir Med. 2016;111:21-29. doi:10.1016/j.rmed.2016.01.003

5. Kolbeck R, Kozhich A, Koike M, et al. MEDI-563, a humanized antiIL-5 receptor alpha $\mathrm{mAb}$ with enhanced antibody-dependent cellmediated cytotoxicity function. J Allergy Clin Immunol. 2010;125 (6):1344-1353.e1342. doi:10.1016/j.jaci.2010.04.004

6. FitzGerald JM, Bleecker ER, Nair P, et al. Benralizumab, an antiinterleukin-5 receptor $\alpha$ monoclonal antibody, as add-on treatment for patients with severe, uncontrolled, eosinophilic asthma (CALIMA): a randomised, double-blind, placebo-controlled phase 3 trial. Lancet. 2016;388(10056):2128-2141. doi:10.1016/S0140-6736 (16)31322-8

7. Goldman M, Hirsch I, Zangrilli JG, Newbold P, Xu X. The association between blood eosinophil count and benralizumab efficacy for patients with severe, uncontrolled asthma: subanalyses of the Phase III SIROCCO and CALIMA studies. Curr Med Res Opin. 2017;33:126.

8. Nair P, Wenzel S, Rabe KF, et al. Oral glucocorticoid-sparing effect of benralizumab in severe asthma. $N$ Engl J Med. 2017;376(25):24482458. doi:10.1056/NEJMoa1703501

9. Bleecker ER, FitzGerald JM, Chanez P, et al. Efficacy and safety of benralizumab for patients with severe asthma uncontrolled with highdosage inhaled corticosteroids and long-acting $\beta_{2}$-agonists (SIROCCO): a randomised, multicentre, placebo-controlled phase 3 trial. Lancet. 2016;388(10056):2115-2127. doi:10.1016/S0140-6736 (16)31324-1
10. Tetteh EK, Morris S, Titcheneker-Hooker N. Discrete-choice modelling of patient preferences for modes of drug administration. Health Econ Rev. 2017;7(1):26. doi:10.1186/s13561-017-0162-6

11. Stoner KL, Harder H, Fallowfield LJ, Jenkins VA. Intravenous versus subcutaneous drug administration: which do patients prefer? A systematic review. Patient. 2014;8:145-153. doi:10.1007/s40271-0140075-y

12. Scalone L, Sarzi-Puttini P, Sinigaglia L, et al. Patients', physicians', nurses', and pharmacists' preferences on the characteristics of biologic agents used in the treatment of rheumatic diseases. Patient Prefer Adherence. 2018;12:2153-2168. doi:10.2147/PPA.S168458

13. Somerville L, Bardelas J, Viegas A, D'Andrea P, Blogg M, Peachey G. Immunogenicity and safety of omalizumab in pre-filled syringes in patients with allergic (IgE-mediated) asthma. Curr Med Res Opin. 2014;30(1):59-66. doi:10.1185/03007995.2013.844115

14. Ferguson GT, Mansur AH, Jacobs JS, et al. Assessment of an accessorized pre-filled syringe for home-administered benralizumab in severe asthma. $J$ Asthma Allergy. 2018;11:63-72. doi:10.2147/JAA. S157762

15. Martin UJ, Fuhr R, Forte P, et al. Comparison of autoinjector with accessorized prefilled syringe for benralizumab pharmacokinetic exposure: AMES trial results. J Asthma. 2019. doi:10.1080/ 02770903.2019.1663428. [Epub ahead of print].

16. Juniper EF, Bousquet J, Abetz L, Bateman ED. GOAL committee. Identifying 'well-controlled' and 'not well-controlled' asthma using the Asthma Control Questionnaire. Respir Med. 2006;100(4):616621. doi:10.1016/j.rmed.2005.08.012

17. Ferguson GT, FitzGerald JM, Bleecker ER, et al. Benralizumab for patients with mild to moderate, persistent asthma (BISE): a randomised, double-blind, placebo-controlled, phase 3 trial. Lancet Respir Med. 2017;5(7):568-576. doi:10.1016/S2213-2600(17)30190-X

18. Callis Duffin K, Bagel J, Bukhalo M, et al. Phase 3, open-label, randomized study of the pharmacokinetics, efficacy and safety of ixekizumab following subcutaneous administration using a prefilled syringe or an autoinjector in patients with moderate-to-severe plaque psoriasis (UNCOVER-A). J Eur Acad Dermatol Venereol. 2017;31 (1):107-113. doi:10.1111/jdv. 13768

19. Ramael S, Van Hoorick B, Tiessen R, et al. Similar pharmacokinetics of the adalimumab (Humira ${ }^{\circledR}$ ) biosimilar BI 695501 whether administered via subcutaneous autoinjector or prefilled syringe (VOLTAIRE $^{\circledR}$-AI and VOLTAIRE ${ }^{\circledR}-\mathrm{TAI}$ ): phase 1 , randomized, open-label, parallel-group trials. Rheumatol Ther. 2018;5(2):403421. doi:10.1007/s40744-018-0119-1

20. Sheikh SZ, Hammer AE, Fox NL, et al. Evaluation of a novel autoinjector for subcutaneous self-administration of belimumab in systemic lupus erythematosus. Int J Clin Pharmacol Ther. 2016;54 (11):914-922. doi: 10.5414/CP202623

21. Cohen S, Klimiuk PA, Krahnke T, Assudani D. Successful administration of BI 695501, an adalimumab biosimilar, using an autoinjector (AI): results from a phase II open-label clinical study $\left(\right.$ VOLTAIRE $^{\circledR}$ RL). Expert Opin Drug Deliv. 2018;15(6):545-548. doi:10.1080/ 17425247.2018.1472572

22. Stauffer VL, Sides R, Lanteri-Minet M, et al. Comparison between prefilled syringe and autoinjector devices on patient-reported experiences and pharmacokinetics in galcanezumab studies. Patient Prefer Adherence. 2018;12:1785-1795. doi:10.2147/PPA.S170636

23. Vermeire S, D'Heygere F, Nakad A, et al. Preference for a prefilled syringe or an auto-injection device for delivering golimumab in patients with moderate-to-severe ulcerative colitis: a randomized crossover study. Patient Prefer Adherence. 2018;12:1193-1202. doi:10.2147/PPA.S154181

24. Heinemann L, Beals JM, Malone J, et al. Concentrated insulins: history and critical reappraisal. J Diabetes. 2018;11:292-300. doi:10.1111/1753-0407.12861 


\section{Publish your work in this journal}

The Journal of Asthma and Allergy is an international, peer-reviewed open-access journal publishing original research, reports, editorials and commentaries on the following topics: Asthma; Pulmonary physiology; Asthma related clinical health; Clinical immunology and the immunological basis of disease; Pharmacological interventions and new therapies. The manuscript management system is completely online and includes a very quick and fair peer-review system, which is all easy to use. Visit http://www.dovepress.com/testimonials.php to read real quotes from published authors.

Submit your manuscript here: https://www.dovepress.com/journal-of-asthma-and-allergy-journal 